\title{
Management of Obstructive Urolithiasis in Male Buffalo Calf
}

\author{
Sanjay Kumar ${ }^{\text {* }}$, Shivinder Pal $^{2}$, Mukesh Gupta ${ }^{3}$, Shivraj Singh Kaleka \\ and Krishan Kumar Nimesh
}

\author{
${ }^{1}$ Surgical Specialist, Veterinary Polyclinic, Sangrur, Punjab, India \\ ${ }^{2}$ Veterinary Pathologist, Veterinary Polyclinic, Sangrur, Punjab, India \\ ${ }^{3}$ Vety. Officer, CVH Ubhawal, Sangrur, Punjab, India \\ ${ }^{4}$ Vety. Officer, CVH Gowara, Sangrur, Punjab, India \\ ${ }^{5}$ SeniorVety. Officer, VHF- Hanumangarh Junction, Rajasthan India \\ *Corresponding author
}

\section{A B S T R A C T}

K e y w o r d s
Tube cystostomy,
Buffalo male calf,
Retention of urine,
Obstructive
Urolithiasis
Article Info
Accepted:
18 March 2020
Available Online:
10 April 2020

\section{Keywords}

Tube cystostomy, Buffalo male calf,

Retention of urine

Obstructive

Accepted:

18 March 2020

Available Ontine April 2020

\section{Introduction}

Treatment of obstructive urolithiasis is definitely surgical, once urethral obstruction is complete (Haven et al., 1993, House et al., 1996). The surgical techniques used include penile transaction with urethral fistulation (Misk and Semieka, 2003), cystic catheterization (Hussain and Moulvi, 1986), pelvic urethrotomy (Ravikumar and Shridhar, 2003), percutaneous tube cystostomy (Streeter et al., 2002) and bladder marsupialization (May et al., 1998) with various complications.

Recurrent urolithiasis, calculi at multiple sites, badly damaged urethra, atonic bladder or severe cystitis are the common complications that may ensue in failure of surgical management of obstructive urolithiasis. Tube cystostomy (Williams and White, 1991) provides an alternative surgical technique in the management of obstructive 
urolithiasis. Advantages of the technique include less chance of recurrences, preservation of the reproductive function of the animal, an opportunity for the removal of cystic calculi and simplicity of the technique. Tube cystostomy, though reportedly successful in small ruminants (Ewoldt et al., 2006) is not widely used in large ruminants, especially cattle, as per literature available.

Urolithiasis is defined as the formation of uroliths/calculus in any part of the urinary system. Obstructive urolithiasis is frequently seen in all species of animals but most commonly in buffalo male calves, cow male calves and goat male kids. In bovines, calculi can lodge anywhere in the urinary tract, but the common sites of obstruction are distal part of Sigmoid flexure and glans penis.

Urinary calculi formation is a condition of multifactorial origin, which results from a combination of physiological, nutritional and managemental factors. However, it is mainly attributed to excessive or imbalanced intake of minerals particularly calcium, phosphorus and magnesium (Radostits et al., 2000).

Obstruction of urethra due to calculi or concretions results in retention of urine in urinary bladder and it's over distention. Failure to provide treatment at an early stage often leads to rupture of either urinary bladder or urethra ensuing in rapid deterioration of the condition of the animal. Generally, a surgical procedure is considered successful when obstruction does not recur without accounting for morbidity i.e. urine scald or cystitis (Van Metre et al., 1996; May et al., 1998).

Tube cystostomy technique provides an alternative surgical treatment for perineal urethrotomy and urethrostomy techniques that have poor long term outcome (Haven et al., 1993), because of stricture formation at urethrotomy and urethrostomy site and hence may be useful in preserving the breeding ability in animals.

\section{Case history}

A four-five month old Murrah crossbreed buffalo male calf presented with the history of not having passed urine since five days and being already treated by some of diuretics. Upon examination there was complete cessation of the urination and distension of abdomen noticed. The other clinical signs were unusual posture and trying to urinate. An aspiratory puncture in the peritoneal cavity was made with a syringe which ruled out uroperitoneum, may be due to rupture of the urinary bladder. The pre-operative vitals of the calf was normal before surgery

The buffalo calf was clinically examined for rectal temperature, general body condition, colour of mucous membrane, heart rate, respiration rate and status of bladder (intact or ruptured) by abdominocentesis and ultrasonography.

Rectal temperature, General body condition, Colour of mucous membrane, Heart rate,

Respiration rate and

Status of bladder (intact or ruptured) by abdominocentesis and ultrasonography.

\section{Anaesthesia}

The calf was infiltrated with $2 \%$ lignocaine injection by lumbosacral route (epidural) and line infiltration at the site of incision.

\section{Restraining and preparation of animal}

The calf was secured on the operation table in right lateral recumbency with flexed position to expose the left ventral caudal abdomen for 
paramedian approach (Fig. 1). The left hind limb tied by bending anteriorly on the hock joint, for exposing the surgical area clearly. Left side of the abdomen near to inguinal region was prepared for surgery by scrubbing.

The animal was prepared for aseptic surgery by shaving and scrubbing of the surgical site i.e. left caudal paramedian abdomen by $2 \%$ cetrimide solution as per standard procedure (Fig. 2). The site of incision was painted with povidone iodine lotion.

\section{Surgical technique}

\section{Tube cystostomy}

A 4-6 cm long incision was made lateral and parallel to the penile urethra in the left prepubic region. Parallel small skin incision was made on the lateral aspect of preputial orifice and a straight artery forceps was passed from the posterior incision site to anterior one to make a subcutaneous tunnel parallel to the penis, and then with the help of artery forceps's tip of Foley's catheter was passed through the subcutaneous tunnel up to the site of posterior incision (Fig. 6).

Peritoneal cavity was entered by separating the abdominal muscles and incising the peritoneum. Then urinary bladder was located after separating the subcutaneous tissue and muscle by blunt incision.

The status of urinary bladder was ruptured (Fig. 4 and 5) hence cystorraphy done after debridement and washed with normal saline to remove concretion sand cystic calculi. Foley's catheters was placed within the urinary bladder (Fig. 7)

The urine outflow was observed through the Foley's catheter after the bulb of the catheter was inflated with $15-20 \mathrm{ml}$ normal saline solution (Fig. 8), as to keep the catheter inside the lumen against the urinary bladder wall. After fixation of Foley's catheter, the abdominal incision was closed using standard procedure. The part of the catheter remaining outside the body (Fig. 9) was stabbed sutured to the abdominal skin.

\section{Post-operative care}

The surgical wound was dressed with povidone-iodine solution and fly repellant spray twice daily till the healing of the wound. Broad spectrum antibiotic Inj. Cetraxione and sulbactum @ 10mg perkg B. Wt. for 7 days. Analgesic Inj. Maloxicam @ $5 \mathrm{mg}$ per $\mathrm{kg} \mathrm{B.Wt}$. for 5 days and Tab. ammonium chloride @200mg per kg B.Wt. BID daily for 1 month (Tamilmahan et al., 2014) and Tab. Gut-boost 1 bid (@a product of Zenegal neutraceutical) for 7 days to prevent anorexia.

After 10-12 days of surgery, dribbling of urine was noticed through urethra (Fig. 10), then opening of Foley's catheter from where urine comes out was tied by a tread or some clamp for 1-2 hours daily to make pressure in the urinary bladder and the period of blocking of Foley's catheter was increased gradually.

When free flow of urine was observed from the urethra (Fig. 3), then the Foley's catheter was tied with thread permanently for 2-3 days. If there is free flow of urine during this period, the Foley's catheter was removed by deflating the balloon (Fig. 11).

Urolithiasis equally occurs both in male and female animals, but obstruction is not generally caused in the female urethra due to the short length and flexible lumen of the urethra as tunica albuginea is not present over the female urethra (Radostits et al., 2000) and sigmoid flexure is only present in the urethra of male ruminants which is the main site of obstruction. 
This could be the reason for obstructive urolithiasis recorded only in male buffalo calves .The concentrate rations are rich in phosphate contents and may predispose them to the urolithiasis (Van Metre, 2004; Singh et

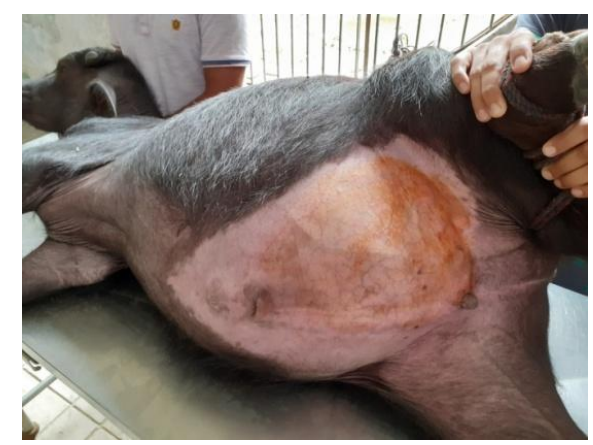

Fig.1 Right lateral recumbency with flexed position to expose the left ventral caudal abdomen

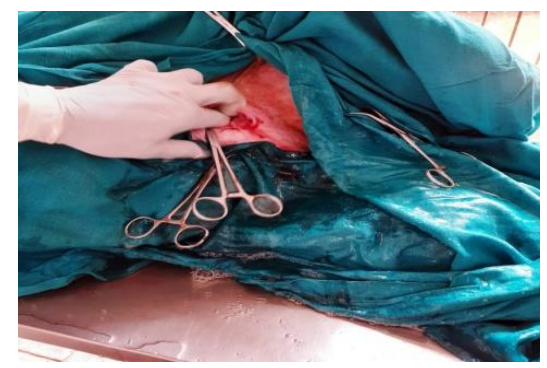

Fig.3 Free Flow of urine after entering the peritoneal cavity

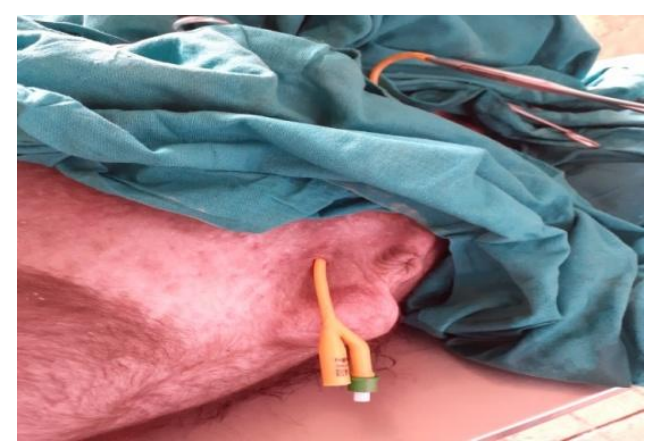

Fig.6 Subcutaneous tunnel parallel to the penis al., 2005). However, in buffalo calves, early weaning, feeding of phosphorus rich diet like wheat bran, rice bran and less water availability might be the reasons for the urolithiasis.

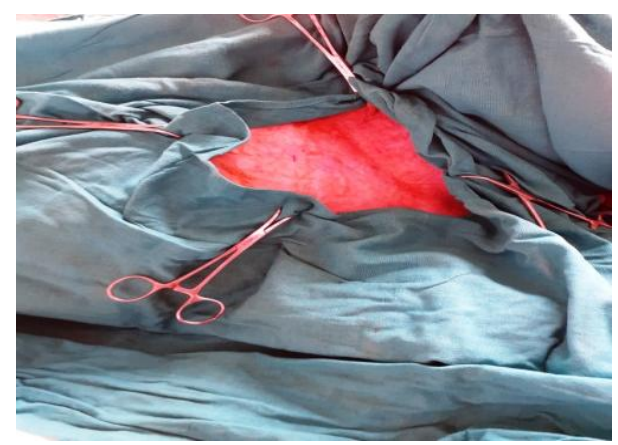

Fig.2 Surgical Site painted with povidone iodine lotion

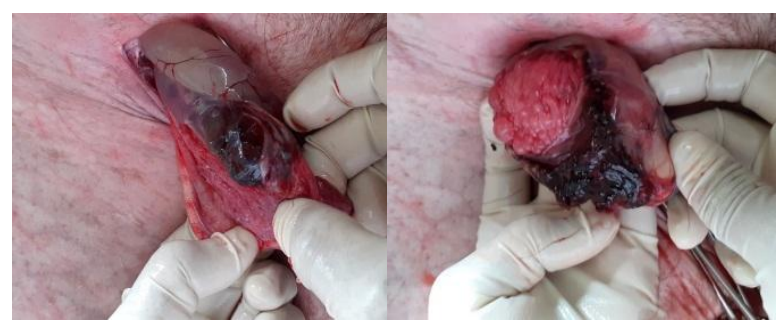

Fig.4\&5 Different conditions of Ruptured urinary Bladder

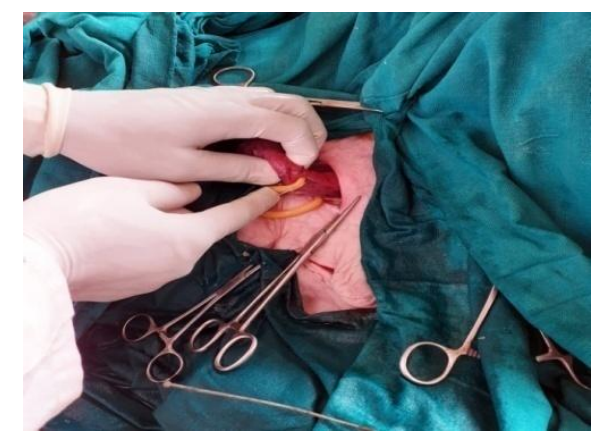

Fig.7 Foley's catheter was placed within the urinary bladder 


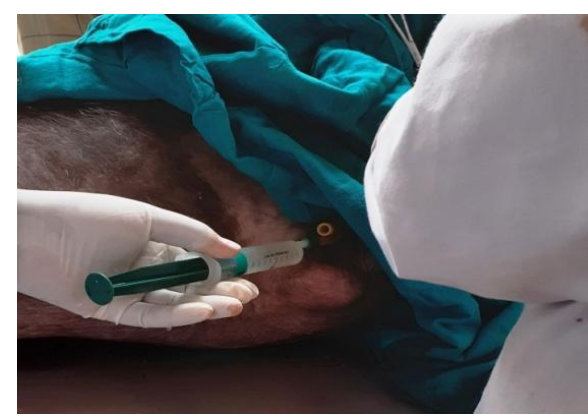

Fig.8 Foley's catheter was inflated normal saline

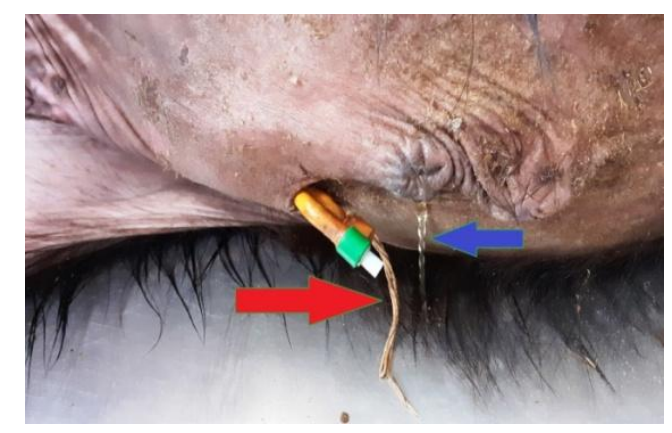

Fig.10 Dribbling of urine through urethra (Blue Arrow) and when opening of Foley's catheter from where urine comes out was tied by a tread

(Red Arrow)

Inappetence and prolonged duration of illness and myocardial asthenia resulting from hyponatraemia and hyperkalaemia in ruminants were suggested the possible cause of increased heart rate in the cases of obstructive urolithiasis. Hyponatraemia and hyperkalaemia as the main causes of the elevated respiration rate in uraemic animals (Radostitis et al., 2000). Bilateral distension of abdomen due to accumulation of urine in peritoneal cavity also causes pressure over diaphragm, which could result in increased and shallow respiration rate.

In conclusion, the occurrence of urolithiasis is higher in uncastrated buffalo male calves in winter season. Tube cystostomy is simple, less time consuming, may be a choice of

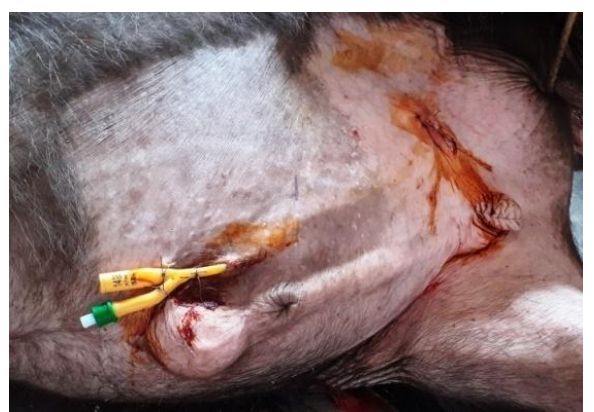

Fig.9 Part of the Foley's catheter remaining outside the body was stabbed sutured to the abdominal skin

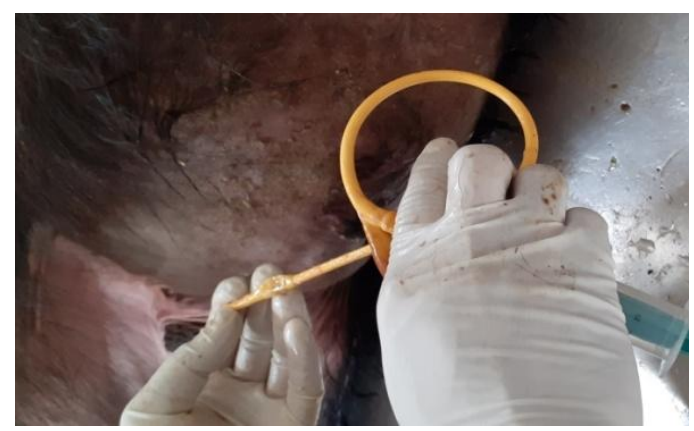

Fig.11 Foley's catheter was removed by deflating the ballon

treatment for the management of the obstructive urolithiasis in buffalo male calves with or without ruptured urinary bladder. Ammonium chloride could be useful in the dissolution of urethral concretions.

\section{References}

Ewoldt, J. M., D. E. Anderson, M. D. Miesner and W. J. Saville (2006): Short-and long-term outcome and factors predicting survival after surgical tube cystostomy for treatment of obstructive urolithiasis in small ruminants. Vet. Surg. 35, 417-422.

Haven, M. L., K. F. Browman and T. A. Engle (1993): Surgical management of urolithiasis in small ruminants. Cornell 
Vet. 83, 47-55.

House, J.K., B.P. Smith and L.W. George (1996). Obstructive urolithiasis in ruminants: Medical treatment and urethral surgery. Compendium Continuing Edu. Pract. Vet. 18, 317328.

Hussain, S. S. and B. A. Moulvi (1986): Treatment of ruptured urinary bladder in a male calf: a case report. Indian Vet. J. 63, 948-951.

May, K., A. H. D. Moll, L. M. Wallace, R. S. Pleasant and R. D. Howard (1998): Urinary bladder marsupialization for treatment of obstructive urolithiasis in male goats. Vet. Surg. 27, 583-588.

Misk, N. A. and M. A. Semieka (2003): Clinical studies on obstructive urolithiasis in male cattle and buffalo. Assuit Vet. Med. J. 49, 258-274.

Radostits, O. M., D. C. Blood, C. C. Gay and K.W. Hinchcliff (2000): Veterinary Medicine: A Textbook of the Diseases of Cattle, Sheep, Pigs, Goats and Horses. $9^{\text {th }}$ ed., Bailliere Tindall, London, pp. 493-498.

Ravikumar, S. B. and W. B. Shridhar. (2003):
Pelvic urethrotomy through infra-anal approach in a bullock- a case report. Indian Vet. J. 80, 59-60.

Singh, J., A. P. Singh, P. K. Peshin, M. Singh, J. S. Gahlawar (1987): Studies on the effects of bilateral ureter obstruction in calves. Indian Vet. J. 64, 127-131.

Streeter. R.N., K.E. Washburn and C.T. McCauley (2002): Percutaneous tube cystostomy and vesicular irrigation for treatment of obstructive urolithiasis in a goat. J. Am. Vet. Med. Assoc. 221,546549.

TamilmahanP, Mohsina A, Karthik K, Gopi M, Gugjoo MB, Rashmi and Zama MMS (2014) Tube cystostomy for management of obstructive urolithiasis in ruminants, Veterinary World 7(4): 234-239.

Van metre, D. (2004): Urolithiasis. Farm Animal Surgery. (Fubini, S. L., G. N. Ducharme, Eds.) W. B. Saunders, New York, pp. 534-547.

Williams, J. M. and R. A. S. White (1991): Tube cystotomy in dog and cat. J. Small Anim. Pract. 32, 598-602.

\section{How to cite this article:}

Sanjay Kumar, Shivinder Pal, Mukesh Gupta, Shivraj Singh Kaleka and Krishan Kumar Nimesh. 2020. Management of Obstructive Urolithiasis in Male Buffalo Calf. Int.J.Curr.Microbiol.App.Sci. 9(04): 2230-2235. doi: https://doi.org/10.20546/ijcmas.2020.904.267 